\title{
OPEN Risk factors for urosepsis in chronic kidney disease patients with urinary tract infections
}

\author{
Zorica Dimitrijevic ${ }^{1,2 \bowtie}$, Goran Paunovic ${ }^{2}$, Danijela Tasic ${ }^{2}$, Branka Mitic ${ }^{1,2}$ \& \\ Dragoslav Basic ${ }^{1,3}$
}

Occurrence of urosepsis is not uncommon following urinary tract infections (UTI). However, there is a lack of evidence explaining the risk factors predisposing to urosepsis in patients with chronic kidney disease (CKD). This retrospective study was undertaken to evaluate the incidence and possible risk factors for urosepsis among patients hospitalized with UTI in a cohort of CKD patients. Patients were divided into the urosepsis group and the non-urosepsis group. Of $\mathbf{4 8 9}$ hospitalized patients with UTI, 70 (14.3\%) acquired urosepsis. Stepwise multivariate logistic regression demonstrated that diabetes, urinary catheter and length of hospital stay $(p<0.001$ for all) were significant independent predictive risk factors for urosepsis in CKD patients with UTI in addition to age, glomerular filtration rate, hydronephrosis, acute kidney injury and E. coli infection ( $p<0.05$ for all). Finally, Klebsiella spp. cases were associated with significantly higher odds for urosepsis than $E$. coli cases (OR: $3.5,95 \% \mathrm{CI}$ : $2.86-7.23, p<0.001$ vs. OR: $1.38,95 \% \mathrm{Cl}: 1.19-3.69, p=0.038$ ). Diabetes, presence of an indwelling urinary catheter, length of hospitalization, and infection with Klebsiella spp were independent risk factors for urosepsis in CKD patients with UTI.

The growing burden of chronic kidney disease (CKD) is a serious public health concern in the twenty-first century. Patients with CKD experience increased morbidity and mortality compared with the non-CKD population, typically from cardiovascular disease. However, infections in people with CKD are a significant source of morbidity and mortality too. The incidence of the commonly seen infectious complications is approximately three times greater among CKD patients than in the general population ${ }^{1}$.

CKD is a risk factor for developing urinary tract infections (UTIs) mostly due to metabolic abnormalities resulting in alterations in primary host defense mechanisms. UTI comprises heterogeneous conditions ranging from mild cystitis, easily treated with oral antibiotics, to life-threatening sepsis and multiple organ failure. Severe or life-threatening infections are usually present as complicated UTI (cUTI) cases. The term complicated urinary tract infection is widely used for an infection that occurs in a patient with a structural or functional abnormality of the genitourinary tract that impedes urine flow or in the presence of the underlying diseases ${ }^{2}$. Therefore, all UTIs in patients with CKD are considered complicated. Urosepsis refers to a clinically manifested severe infection of the urinary tract. It is assumed that ascending UTI from the bladder to the kidney, with resultant bacteremia, is the primary cause of urosepsis. Urosepsis in adults comprises approximately $25 \%$ of all sepsis cases following an episode of $\mathrm{CUTI}^{3}$. Gram-negative rods (75-85\%) are most commonly associated with the abovementioned condition, while gram-positive organisms are less frequently (15\%) involved. While urosepsis patients have the lowest mortality rate among patients suffering from all causes of sepsis, urosepsis may still lead to mortality rates of $25 \%$ to $60 \%$ in different patient groups ${ }^{4}$. However, not all patients with cUTI will develop urosepsis. A number of studies have analyzed the risk factors for sepsis in different patients population ${ }^{5-11}$, but not in CKD patients with UTI. Therefore, this study was undertaken to evaluate the incidence and possible risk factors for urosepsis among patients hospitalized with UTI in a cohort of CKD patients attending the nephrology clinic.

\section{Methodology}

This retrospective observational study was conducted at the Clinic of Nephrology, Clinical Center Nis, Serbia. The Ethics Committee of the University Clinical Center Nis approved the study (approval number 12734) and the need for informed consent was waived, as no additional blood sampling was needed and routine patient care was not modified. The study has been performed in accordance with the Declaration of Helsinki. We analyzed

${ }^{1}$ Faculty of Medicine, University of Nis, Bulevar Dr. Z. Djindjica 81, 18000 Niš, Serbia. ${ }^{2}$ Clinic for Nephrology, Clinical Center Nis, Bulevar Dr. Z. Djindjica 48, 18000 Niš, Serbia. ${ }^{3}$ Clinic for Urology, Clinical Center Nis, Bulevar Dr. Z. Djindjica 48, 18000 Niš, Serbia. ${ }^{\varpi}$ email: zorica_mdimitrijevic@yahoo.com 
data from 489 CKD patients diagnosed and hospitalized with UTI from January 2017 to December 2019. UTI criteria were based on clinical symptoms and laboratory diagnosis, including dysuria with bacterial isolation of more than $10^{5}$ colony forming units (CFU)/mL. Urosepsis refers to sepsis caused by infection of the urogenital tract $^{4}$ with the isolation of the same pathogen from urine and blood cultures. In this study, we complied with the criteria of $\mathrm{ACCP} / \mathrm{SCCM}^{12}$. CKD is defined as kidney damage or glomerular filtration rate (GFR) $<60 \mathrm{~mL} /$ $\mathrm{min} / 1.73 \mathrm{~m}^{2}$ for three months or more, irrespective of cause $\mathrm{e}^{13}$.

Patients were then divided into two groups: cases with UTI at time of admission or during hospitalization but without urosepsis and cases with urosepsis (urosepsis group consisting of patients who were diagnosed as urinary tract infection with two or more clinical findings of systemic inflammation response syndrome (SIRS), including temperature $>38.0^{\circ} \mathrm{C}$ or $<36.0^{\circ} \mathrm{C}$; heart rate $>90 / \mathrm{min}$; respiration rate $>20 / \mathrm{min}$ or $\mathrm{PaCO}_{2}<32 \mathrm{mmHg}$ or less; WBC count $>12,000 / \mathrm{mm}^{3},<4000 / \mathrm{mm}^{3}$ ).

We analyzed patients' pre-existing medical conditions with the potential to contribute to UTI-induced urosepsis. The demographic data, comorbidities, baseline renal function, the presence of indwelling urinary tract catheter prior to UTI, previous antibiotics use, bacterial isolates, length of hospital stay, and the occurrence of acute kidney injury (AKI) during hospitalization were recorded in all cases. Patients with clinical signs of UTI but a positive blood culture indicating another focus were excluded. An abdominal ultrasound was also done on arrival at the nephrology clinic. An abdominal CT or other radiologic examinations were performed at the discretion of the treating nephrologist. A diagnosis of urinary tract obstruction was made when there was either hydronephrosis or obstructive uropathy. Hydronephrosis was defined as a dilation of the renal pelvis and calyces proximal to the point of obstruction ${ }^{14}$. Obstructive uropathy refers to narrowing or blockage of the urine flow caused by functional or structural disorders anywhere from the tip of the urethra to the renal pelvis, which increases the pressure proximal to the site of the obstruction ${ }^{15}$.Ceftriaxone $1-2 \mathrm{~g} \mathrm{IV} / 24 \mathrm{~h}$ was initially prescribed as an empirical antibiotic to all patients with CKD and UTI. For patients with penicillin allergies, fluoroquinolones were an option; as all patients suffered from CKD, we refrained from aminoglycosides. The definitive antimicrobial therapy was determined by the drugs administrated after the microbiological data were available. Finally, antimicrobial susceptibility testing was performed for all urine and blood specimens.

Statistics. Parametric data are presented as mean \pm standard deviation and non-parametric data as median (inter-quartile range). T-test or Mann-Whitney test was used to correlate continuous data depending on whether data were parametric or non-parametric. The differences in categorical variables between groups were analyzed by the chi-square test. Univariable analyses were done to identify parameters that were associated with the occurrence of urosepsis. Consequently, the multivariable logistic regression model was generated to identify demographic and other parameters independently associated with urosepsis occurrence. Demographic parameters and comorbid conditions that were found to be associated with urosepsis on univariable analysis were incorporated in the model as covariates. Adjusted odds ratios and $95 \%$ confidence intervals were then determined for each covariate in the model to identify independent risk factors for the development of urosepsis. The predictive performance of single independent predictors and combined independent predictors of risk for urosepsis was further assessed by plotting receiver operating characteristic (ROC) curves and calculating sensitivity, specificity, and area under the curve (AUC)s with 95\% confidence intervals (CIs).

\section{Results}

A total of 489 CKD patients with UTI, 229 (46.8\%) males and 260 (53.2\%) females, were enrolled for final analysis. The median age of the patients was 59.5 years (IQR 26-81). The demographic and clinical characteristics of UTI patients with and without urosepsis are shown in Table 1.

Patients with UTI who developed urosepsis were older than those who did not $(67.2 \pm 14.1$ years old versus $52 \pm 19.4$ years old, $p<0.001)$. Higher prevalence of diabetes $(78.5 \%$ versus $22.3 \%, p<0.001)$, pelvic malignancy (51.4\% versus $7.63 \%, p<0.001)$, indwelling urinary catheter $(71.4 \%$ versus $9.31 \%, p<0.001)$ and prolonged hospitalization $(19.4 \pm 25.3$ versus $6.8 \pm 5.6, p<0.001)$ were observed in the urosepsis group as compared to those without. In addition, lower GFR (20.3 \pm 10.3 versus $31.6 \pm 9.1)$, occurrence of AKI (100\% versus $69.2 \%)$, the presence of hydronephrosis $(57.4 \%$ versus $24.4 \%, p=0.036)$ nephrostomy tube $(14.2 \%$ versus $2.62 \%, p=0.025)$ and prior antibiotics use $(62.8 \%$ versus $30.1 \%, p=0.039)$ were also associated with urosepsis. The presence of hypertension and previous cerebrovascular insult (CVI) were similar in both groups. We did not observe gender differences between the two groups of patients.

This was the first episode of UTI for 251 (51.3\%) patients, while the other $238(48.7 \%)$ have had previous episodes. Most patients with urosepsis have a history of recurrent UTI (74.2\% versus $44.4 \%)$. Table 2 shows the distribution of pathogens per two patients groups. E.coli was most commonly identified in CKD UTI patients, responsible for $46.8 \%$ of cases, followed by Klebsiella spp with $11.2 \%$ of UTI cases. On the other hand, in urine samples from patients with urosepsis, Klebsiella spp $(21.4 \%$; $15 / 70)$ was more frequently isolated, with gramnegative bacilli account for nearly $66 \%$ of the cases of urosepsis. Interestingly, gram-positive Enterococcus sppwas the third most frequent cause of urosepsis in our patients' group (15.7\% of cases). Most urosepsis (94.3\%) were monomicrobial.

The rates of susceptibility to 13 selected antimicrobial agents against gram-negative bacilli and gram-positive cocci are summarized in Table 3.Considering the antibiotics sensitivity, we found that most of the gram-negative bacteria were susceptible in varying degrees to cefepime, carbapenems, aminoglycosides, and levofloxacin. The ranges of resistance for gram-positive isolates were from 0 to 92 .

Univariate logistics analysis showed that age $(p=0.007)$, diabetes $(p<0.001)$, baseline GFR $(p=0.031)$, previous episodes of UTI $(p=0.038)$, pelvic malignancy $(p=0.034)$, presence of indwelling catheter $(p<0.001)$, hydronephrosis $(p=0.042)$, AKI $(p=0.046)$, duration of hospitalisation $(p<0.001)$, infections with $E$. coli $(p=0.026)$, 


\begin{tabular}{|c|c|c|c|c|}
\hline \multirow[b]{2}{*}{ Characteristics } & \multirow[b]{2}{*}{ All $(n=489)$} & \multicolumn{2}{|c|}{ Presence of urosepsis } & \multirow[b]{2}{*}{$p$ value } \\
\hline & & $\begin{array}{l}\text { Yes } \\
n=70\end{array}$ & $\begin{array}{l}\text { No } \\
n=419\end{array}$ & \\
\hline Age & $59.5 \pm 21$ & $67.2 \pm 14.1$ & $52 \pm 19.4$ & $<0.001$ \\
\hline \multicolumn{5}{|l|}{ Gender } \\
\hline Female & $260(53.2 \%)$ & $38(54.3 \%)$ & $222(52.9 \%)$ & \multirow{2}{*}{ ns } \\
\hline Male & $229(46.8 \%)$ & $32(45.7 \%)$ & $197(47.1 \%)$ & \\
\hline Preexisting diabetes & $109(22.3 \%)$ & $55(78.6 \%)$ & $54(12.9 \%)$ & $<0.001$ \\
\hline Hypertension & $302(61.7 \%)$ & $34(48.5 \%)$ & $268(64 \%)$ & ns \\
\hline Previous CVI & $44(9 \%)$ & $13(18.5 \%)$ & $31(7.4 \%)$ & ns \\
\hline Baseline GFR & $40.2 \pm 18.1$ & $20.3 \pm 10.3$ & $31.6 \pm 9.1$ & \multirow{4}{*}{0.035} \\
\hline GFR 31-60 & $138(28.2 \%)$ & $12(17.1 \%)$ & $126(30.1 \%)$ & \\
\hline GFR $16-30$ & $172(35.17 \%)$ & $18(25.7 \%)$ & $154(36.7 \%)$ & \\
\hline GFR $<15$ & $179(36.6 \%)$ & $36(51.4 \%)$ & $142(33.8 \%)$ & \\
\hline Acute kidney injury & $360(73.6 \%)$ & $70(100 \%)$ & $290(69.2 \%)$ & 0.023 \\
\hline Previous history of UTI & $238(48.6 \%)$ & $52(74.2 \%)$ & $186(44.4 \%)$ & 0.031 \\
\hline Pelvic malignancy & $68(13.9 \%)$ & $36(51.4 \%)$ & $32(7.63 \%)$ & $<0.001$ \\
\hline Indwelling urinary catheter & $89(18.2 \%)$ & $50(71.4 \%)$ & $39(9.31 \%)$ & $<0.001$ \\
\hline Hydronephrosis & $134(27.4 \%)$ & $40(57.4 \%)$ & $94(22.4 \%)$ & 0.036 \\
\hline Nephrostomy tube & $21(4.3 \%)$ & $10(14.2 \%)$ & $11(2.62 \%)$ & 0.025 \\
\hline Renal stone & $90(18.4 \%)$ & $28(40 \%)$ & $92(14.8 \%)$ & 0.054 \\
\hline Prior antibiotics within 3 months & 150 & $44(62.8 \%)$ & $126(30.1 \%)$ & 0.039 \\
\hline Length of hospital stay (days) & $13.2 \pm 18.5$ & $19.4 \pm 25.3$ & $6.8 \pm 5.6$ & $<0.001$ \\
\hline
\end{tabular}

Table 1. Baseline demographic, clinical, and laboratory characteristics of the patients with complicated urinary tract infection. Bold values denote statistical significance at the $p<0.05$ level. CVI cerebrovascular insult, GFR glomerular filtration rate, $n s$ non-significant.

\begin{tabular}{|l|l|l|l|}
\hline \multicolumn{2}{|l|}{$\begin{array}{l}\text { Patients with UTI } \\
\mathbf{n}=\mathbf{4 1 9}\end{array}$} & $\begin{array}{l}\text { Patients with urosepsis } \\
\mathbf{n = 7 0}\end{array}$ & $\boldsymbol{p}$ value \\
\hline Gram-negative rods & $196(39.2 \%)$ & $12(17.1 \%)$ & $<0.001$ \\
\hline Escherichia coli & $47(11.2 \%)$ & $15(21.4 \%)$ & 0.020 \\
\hline Klebsiella spp & $40(9.54 \%)$ & $9(12.8 \%)$ & $\mathrm{ns}$ \\
\hline Enterobacter & $30(7.15 \%)$ & $4(5.7 \%)$ & $\mathrm{ns}$ \\
\hline Proteus spp & $26(6.2 \%)$ & $3(4.3 \%)$ & $\mathrm{ns}$ \\
\hline Acinetobacter & $18(3.6 \%)$ & $3(4.3 \%)$ & $\mathrm{ns}$ \\
\hline Pseudomonas aeruginosa & & & 0.030 \\
\hline Gram-positive cocci & $30(7.15 \%)$ & $11(15.7 \%)$ & 0.036 \\
\hline Enterococcus spp & $12(2.9 \%)$ & $4(5.7 \%)$ & 0.043 \\
\hline Staphyloccocus aureus & $20(4.77 \%)$ & $5(7.1 \%)$ & 0.050 \\
\hline Methicillin-resistant Staphylococcus aureus (MRSA) & $10(2 \%)$ & $4(5.7 \%)$ & \\
\hline Multiple organisms & & & \\
\hline
\end{tabular}

Table 2. Bacterial isolates from urine and blood cultures. $n s$ Non-significant.

Klebsiella spp $(p<0.001)$ and Enterococcus spp $(p=0.045)$, were significantly correlated with a high risk of urosepsis in CKD patients with UTI (Table 4).

In a forward stepwise multivariate logistic regression, diabetes, urinary catheter, length of hospital stay and Klebsiella infection $(p<0.001$, for all) were significant independent predictive factors for increased risk of urosepsis in CKD patients with UTI in addition to age, GFR, hydronephrosis, AKI and E.coliinfection $(p=0.024$, $p=0.028, p=0.040, p=0.048$ and $p=0.038$, respectively).

Independent, most significant predictive factors (diabetes, urinary catheter, length of hospital stay, and infection with Klebsiellaspp) for risk of urosepsis were included in a ROC curve analysis.

The area under receiver operating characteristic curve(AUROC) of DM was 0.61 (95\%CI: 0.56-0.64, $p=0.05$ ) (Fig. 1). However, ROC curve analysis showed that presence of urinary catheter (AUC: $0.79,95 \% \mathrm{CI}$ : $0.70-0.82, p<0.001$ ), infection with Klebsiella spp (AUC: 0.74, 95\%CI: 0.71-0.84, $p<0.001$ ), and duration of hospitalisation (AUC: $0.68,95 \% \mathrm{CI}: 0.60-0.79, p<0.001$ ) could predict increased risk from urosepsis in CKD patients with UTI. In addition, the combination of these three risk factors had a greater predictive value than each individual factor alone (AUC: 0.82 , 95\%CI: 0.79-0.88). 


\begin{tabular}{|c|c|c|c|c|c|c|c|c|c|c|c|c|c|}
\hline & AMP & AMC & CTX & CTAX & CFR & CAZ & CFP & CIP & LVX & MER & IMI & AMI & VAN \\
\hline E.coli & 14.6 & 40.2 & 31.5 & 51.8 & 52.2 & 31.2 & 72.6 & 52 & 73 & 89.4 & 100 & 90 & 1 \\
\hline Klebsiella spp & 4.1 & 31.2 & 9.4 & 18.2 & 14.2 & 16 & 35.2 & 33.2 & 45.3 & 94.2 & 97 & 81 & 3.8 \\
\hline Enterobacter & 6.2 & 16.8 & 35.1 & 53.1 & 55.2 & 26.2 & 60.1 & 42.2 & 50.3 & 76.2 & 100 & 80.2 & 1 \\
\hline Proteus & 13.8 & 26.4 & 46.3 & 28.6 & 37.2 & 36.4 & 56.4 & 52.8 & 60.8 & 98.2 & 100 & 54.2 & 0 \\
\hline Acinetobacter & 0 & 10.2 & 0 & 0 & 0 & 0 & 12.2 & 29.1 & 35.7 & 58 & 53.6 & 45 & 0 \\
\hline Pseudomonas aeruginosa & 0 & 0 & 2 & 5.2 & 8.7 & 45.2 & 46.2 & 30.1 & 33.5 & 58.3 & 48.2 & 76 & 1 \\
\hline Enterococcus faecalis & 53.2 & 58.5 & 12.3 & 10.4 & 11.2 & 2.1 & 15.3 & 50.6 & 58.2 & 46.3 & 45.9 & 40.3 & 91.4 \\
\hline Staphyloccocus aureus & 1 & 1 & 8 & 26.3 & 46.2 & 27.3 & 55.6 & 44.2 & 50.8 & 1 & 1 & 42.2 & 90.3 \\
\hline $\begin{array}{l}\text { Methicillin-resistant Staphylococcus aureus } \\
\text { (MRSA) }\end{array}$ & 22.72 & 18.6 & 1 & 1 & 1 & 1 & 1 & 36.2 & 40.1 & 50.1 & 62.2 & 32 & 100 \\
\hline
\end{tabular}

Table 3. Antibiotics susceptibility patterns of isolated uropathogens (\% sensitivity). AMP Ampicillin, AMC Amoxicillin/clavulanic acid, CTX Cefotaxime, CTAX Ceftriaxone, CFR Cefuroxime, CAZ Ceftazidime, CFP Cefepime, CIP Ciprofloxacin, LVX Levofloxacin, MER Meropenem, IMI Imipenem, AMI Amikacin, VAN Vancomycin, / Not done.

\begin{tabular}{|l|l|l|l|l|l|l|}
\hline \multirow{2}{*}{ Variable } & \multicolumn{3}{|l}{ Univariate analysis } & \multicolumn{3}{l|}{ Multivariate analysis } \\
\cline { 2 - 8 } & OR & $\mathbf{9 5 \%}$ CI & $\boldsymbol{p}$ value & OR & $\mathbf{9 5 \%}$ CI & $\boldsymbol{p}$ value \\
\hline Gender (male/female) & 0.48 & $0.39-1.52$ & ns & & & \\
\hline Age (years) & 1.36 & $1.08-1.99$ & $\mathbf{0 . 0 0 7}$ & 1.46 & $1.24-1.57$ & $\mathbf{0 . 0 2 4}$ \\
\hline Diabetes mellitus & 5.14 & $3.11-7.41$ & $<\mathbf{0 . 0 0 1}$ & 5.02 & $3.20-8.35$ & $<\mathbf{0 . 0 0 1}$ \\
\hline Previous episodes of UTI & 3.70 & $2.24-4.96$ & $\mathbf{0 . 0 3 8}$ & 2.15 & $1.28-4.55$ & ns \\
\hline Baseline GFR & 4.58 & $3.14-7.55$ & $\mathbf{0 . 0 3 1}$ & 5.14 & $4.01-9.66$ & $\mathbf{0 . 0 4 0}$ \\
\hline Pelvic malignancy & 1.62 & $1.27-2.576$ & $\mathbf{0 . 0 3 4}$ & 1.36 & $1.24-2.11$ & ns \\
\hline Indwelling urinary catheter & 2.92 & $1.94-5.52$ & $<\mathbf{0 . 0 0 1}$ & 3.71 & $2.36-4.88$ & $<\mathbf{0 . 0 0 1}$ \\
\hline Hydronephrosis & 4.12 & $1.50-5.71$ & $\mathbf{0 . 0 4 2}$ & 2.58 & $1.71-3.14$ & $\mathbf{0 . 0 4 0}$ \\
\hline Acute kidney injury & 2.66 & $2.17-5.36$ & $\mathbf{0 . 0 4 6}$ & 2.85 & $2.01-3.92$ & $\mathbf{0 . 0 4 8}$ \\
\hline Previous antibiotics treatment & 2.11 & $1.88-2.99$ & ns & & & \\
\hline Length of hospital stay & 3.48 & $2.55-6.89$ & $<\mathbf{0 . 0 0 1}$ & 3.59 & $2.32-5.03$ & $<\mathbf{0 . 0 0 1}$ \\
\hline Infection with E.coli & 2.70 & $2.01-5.20$ & $\mathbf{0 . 0 2 6}$ & 1.38 & $1.19-3.69$ & $\mathbf{0 . 0 3 8}$ \\
\hline Infection with Klebsiella spp & 4.38 & $3.62-9.60$ & $<\mathbf{0 . 0 0 1}$ & 3.50 & $2.86-7.23$ & $<\mathbf{0 . 0 0 1}$ \\
\hline Infection with Enterococcus spp & 2.213 & $1.89-3.78$ & $\mathbf{0 . 0 4 5}$ & 1.22 & $1.01-2.11$ & ns \\
\hline
\end{tabular}

Table 4. Logistic regression model for factors related to urosepsis. Bold values denote statistical significance at the $p<0.05$ level. OR Odds ratio, CI Confidence interval, GFR Glomerular filtration rate, $n s$ Non-significant.

\section{Discussion}

Urinary tract infection and urosepsis represent one of the most demanding problems in medicine. Past studies have shown that the sepsis rate in patients with cUTIs ranges between 20.8 and $32.9 \%$ depending on various underlying conditions ${ }^{16,17}$. Numerous risk factors associated with sepsis have been previously reported among cUTI patients, including older age, female gender, diabetes, immunosuppression, AIDS, anemia, calculus diameter $>2.5 \mathrm{~cm}$, liver cirrhosis, and indwelling urinary catheter use $\mathrm{e}^{17,18}$. To our best knowledge, this is the first study to evaluate risk factors for urosepsis in CKD patients.

It is well known that the incidence of UTI in older adults rises substantially. As reported, bacteriuria is present in at least $20 \%$ of women and $10 \%$ of men aged 65 or older $^{19}$. Consistent with the previous studies ${ }^{20,21}$, older age is also associated with a higher risk of urosepsis in our study group, with an odds ratio of 1.36 incremented each year in the univariate logistic regression model. The elderly with CKD are particularly prone to sepsis due to their many comorbidities, repetitive and extended hospitalizations, inadequate capacity to fight infections, and functional limitations associated with aging. Multiple protective mechanisms decrease along with age. These include cell-mediated immunity, fecal and urinary incontinence-related contamination, the occurrence of obstructive uropathy, urethral instrumentation, and catheterization in addition to hormone-related antibacterial factors reduction in the prostate and vagina. As a result of anatomical differences, the complexity of UTI in men and women is significantly different. However, we showed that both genders shared similar risk factors, and no differences in urosepsis incidence were seen between them.

The prevalence of diabetes in our study was $22.3 \%$. A variety of causes, including glycosuria, neutrophil dysfunction, and increased bacterial adherence of bacteria to uroepithelial cells, are the reasons for the more frequent occurrence of UTI in diabetic patients. Age, metabolic control, diabetic nephropathy, autonomic neuropathy, and vascular complications are additional factors that increase the risk of UTI in diabetes ${ }^{22}$. Diabetes mellitus is one of the most common comorbidities in patients with sepsis, possible due to impaired immune 


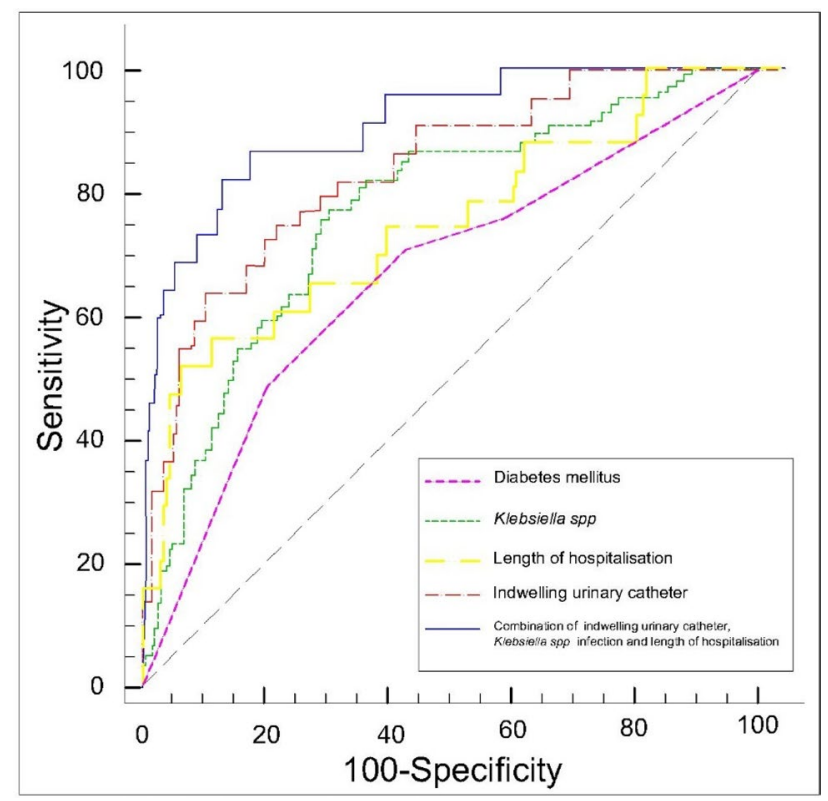

Figure 1. Receiver operating curve (ROC) for urosepsis risk score prediction in CKD patients with UTI.

responses $^{23}$. In our urosepsis group, $78.6 \%$ of patients had pre-existing diabetes. This finding is in agreement with previous studies ${ }^{24,25}$.

We demonstrated that lower baseline GFR significantly contributes to the occurrence of urosepsis. The hospitalization rate due to septicemia in patients with CKD appears to be 3-to fourfold higher than in patients without $\mathrm{CKD}^{26}$. Nevertheless, it is unclear whether this observation is attributable just to the consequences of CKD or should be explained by the older age and cumulative comorbidity burden in patients with CKD. Renal dysfunction may be an indicator of other conditions that increase susceptibility to infection. The urologic diseases may partly explain the increased risk of bloodstream infection in CKD settings owing to infections from urinary tract sources. Alternatively, this relationship could be elucidated by several consequences of CKD that may contribute to infection, including malnutrition, chronic inflammation, retention of uremic toxins, and metabolic abnormalities ${ }^{27}$. Likewise, abnormalities in neutrophil and lymphocyte functions observed in CKD patients indicate that immune function impairment possibly contributes to infection susceptibility in the setting of CKD ${ }^{28}$.

Acute UTI can cause an abrupt decline of renal function, especially in urinary tract obstruction. Several studies have suggested that AKI is not a prevalent complication among patients with acute pyelonephritis ${ }^{29,30}$. In this study, the incidence of AKI was very high (73.6\%) compared to $12.3 \%$ in Hsiao et al. study ${ }^{31}$. A likely explanation is that all our patients had pre-existing CKD and more associated comorbidities that additionally contribute to AKI.

Any obstructed urinary drainage system has the potential to be infected. Urosepsis is frequently provoked by obstruction of the upper urinary tract, with urolithiasis being the most common cause. In Liang et al. study, $14.3 \%$ of patients had obstructive lesions that provoked urosepsis ${ }^{32}$. More than half of the urosepsis patients had hydronephrosis in our study group, and $14.2 \%$ had a nephrostomy tube. Percutaneous nephrostomy (PCN) tubes are the most reliable method for draining obstructed kidneys and preventing acute kidney injury. Still, PCN tubes can provoke infective complications, such as pyelonephritis, which have the potential to progress to urosepsis. Sepsis occurs in $1.3 \%$ to $1.8 \%$ of patients with PCN tubes ${ }^{33}$.

Catheter-associated urinary tract infection represents the most common healthcare-associated infection globally, with a fourfold increased risk of UTI compared to those without a urinary catheter ${ }^{18,34}$. Placement of an indwelling urinary catheter poses a risk of developing bacteriuria of 3-10\% per day, with bacteriuria being considered ubiquitous on the 30 th day ${ }^{35,36}$. In our multivariate regression analysis, the presence of the urinary catheter was an independent predictor of urosepsis, and the ROC curve was more significant than with duration of hospitalization and Klebsiella infection. So, the best way to avoid catheter associated-UTI and consecutive urosepsis is to place a urinary catheter only when strictly necessary, as indicated by international guidelines, and remove it early on.

Consistent with the study of Chan et al. ${ }^{37}$, duration of hospitalization was also identified as an independent risk factor for urosepsis among our patients' population. Prolonged hospitalizations are associated with a risk of infection, especially in those with multiple comorbidities ${ }^{38}$. Although we have shown that longer hospital stay increases the odds for a urosepsis, we could not distinguish whether urosepsis was the cause of longer hospitalization or vice-versa. It is possible that the more extended hospital stay in these cases was a consequence of the infection, requiring a longer hospitalization.

The most common uropathogens in our study were E. coli (46.8\%), Klebsiella spp (11.2\%), and Enterococcus $\operatorname{spp}(9.54 \%)$. It confirms the previous findings indicating that $E$. coli is the predominant etiological agent of UTI in the general population ${ }^{39}$ and the CKD patients ${ }^{40}$. However, the most causative agent of urosepsis in our study 
group was Klebsiella spp, responsible for $21.4 \%$ of the cases. This result is different from the study results conducted from 2003 to 2013, which demonstrated that the overall prevalence of E.coli as a cause of urosepsis was 43\%, followed by Enterococcus spp. (11\%), Klebsiella spp. (10\%) and Pseudomonas aeruginosa (10\% $)^{41}$. Similar results were obtained by other authors ${ }^{42-44}$. Nevertheless, the aforementioned studies were conducted in the general population, not in CKD patients.

It is well recognized that Klebsiella is an important pathogen in hospital settings, often associated with nosocomial infections, with a significant proportion of resistance to multiple drugs ${ }^{45}$. The hospitalized, immunocompromised patients with significant underlying diseases are the main targets of this pathogenic bacteria. Consequently, there is a natural tendency to hospitalize CKD UTI cases of greater severity.

An additional explanation for a high proportion of Klebsiella urosepsis in our study group is that $71.4 \%$ of our urosepsis population had indwelling urinary catheters. This finding supports recent studies demonstrating that Klebsiella infection was more associated with a urinary catheter than E. coli ${ }^{46,47}$. Unfortunately, this high proportion of Klebsiella infections in our study group may also be explained by healthcare-associated transmission.

A specific 'resistance profile' to antimicrobial therapy can challenge the initial approach and the management, increasing the risk of treatment failure. Widespread use of broad-spectrum antibiotics in hospitalized patients has led to both increased carriage of Klebsiella and, subsequently, the development of multidrug-resistant strains; the empirically administered antibiotic Ceftriaxone did not cover the Klebsiella spectrum (resistance rate 81.8\%), and therefore, these infections progressed to urosepsis.

Finally, Enterococcus spp was the third commonest causative agent for urosepsis. Enterococcus provokes a robust infection in the catheter-containing bladder, in the kidneys, and on the catheter material itself, where it forms a biofilm that promotes persistent infection in the face of intense catheter-driven inflammation. Recent epidemiological studies have detected that enterococci were the most commonly isolated gram-positive bacteria from catheter-associated urinary tract infections ${ }^{48}$ and are amongst the predominant pathogens isolated from polymicrobial communities on the surface of indwelling urinary catheters ${ }^{49}$. To limit this infection, urinary catheters should only be used if clinically indicated in CKD patients.

Our study had several limitations. The most important is the retrospective design, which is associated with potential biases. The study was conducted in a single center, reflecting the possibility of institutional bias either in the selection of patients or routine medical practices. Additionally, we employed older sepsis and septic shock definitions instead of the recently introduced SOFA criteria ${ }^{50}$.

\section{Conclusion}

The rate of urosepsis in CKD patients with UTI was 14.3\%. Underlying diseases such as diabetes, presence of urinary catheters, length of hospitalization, and infection with Klebsiella spp were independent risk factors for sepsis in CKD patients with UTI. Given the vast number of incidences of UTI, the findings of this study can assist in identifying at-risk patients and guide appropriate care to reduce the incidence of urosepsis further. In addition, understanding the bacteria involved in the development of urosepsis is a fundamental part of developing successful antibiotic treatment. With the high prevalence of Klebsiella infections, healthcare professionals should be aware of the precautions obliged to ensure the minimal transmission of nosocomial infection.

Received: 22 February 2021; Accepted: 29 June 2021

Published online: 13 July 2021

\section{References}

1. Naqvi, S. B. \& Collins, A. J. Infectious complications in chronic kidney disease. Adv. Chronic Kidney Dis. 13, 199-204 (2006).

2. Lichtenberger, P. \& Hooton, T. M. Complicated urinary tract infections. Curr. Infect. Dis. Rep. 10, 499-504 (2008).

3. Wagenlehner, F. M. et al. Diagnosis and management for urosepsis: Items in urosepsis. Int. J. Urol. 20, 963-970 (2013).

4. Wagenlehner, F. M. E., Pilatz, A., Naber, K. G. \& Weidner, W. Therapeutic challenges of urosepsis. Eur. J. Clin. Invest. 38, 45-49 (2008).

5. Peach, B. C. et al. Risk factors for urosepsis in older adults: a systematic review. GerontolGeriatr Med. 2, 2333721416638980 (2016).

6. Wang, S. et al. Risk factors for urosepsis after minimally invasive percutaneous nephrolithotomy in patients with preoperative urinary tract infection. Biomed Res Int. 2, 1354672 (2020).

7. Liu, J. et al. Risk factors and prediction model of urosepsis in patients with diabetes after percutaneous nephrolithotomy. $B M C$ Urol. 28, 74 (2021).

8. Siritip, N. et al. Epidemiology, risk factors, and outcome of bloodstream infection within the first year after kidney transplantation. Am. J. Med. Sci. 361, 352-357 (2021)

9. Qiang, X. H., Yu, T. O., Li, Y. N. \& Zhou, L. X. Prognosis risk of urosepsis in critical care medicine: a prospective observational study. Biomed. Res. Int. 2016, 9028924 (2016).

10. Abou Dagher, G. et al. Sepsis in hemodialysis patients. BMC Emerg. Med. 15, 30 (2015).

11. Powe, N. R., Jaar, B., Furth, S. L., Hermann, J. \& Briggs, W. Septicemia in dialysis patients: incidence, risk factors, and prognosis. Kidney Int. 55, 1081-1090 (1999).

12. Bone, R. C. et al. Definitions for sepsis and organ failure and guidelines for the use of innovative therapies in sepsis. The ACCP/ SCCM Consensus Conference Committee. American College of Chest Physicians/Society of Critical Care Medicine. Chest 101, 1644-1655 (1992).

13. Levey, A. S. et al. The definition, classification, and prognosis of chronic kidney disease: a KDIGO Controversies Conference report. Kidney Int. 80, 17-28 (2011).

14. Kumar, V., Abbas, A. K., Fausto, N. \& Aster, J. C. Robbins and Cotran pathologic basis of disease (8th ed. Philadelphia PA) 960-962 (Elsevier Saunders, 2010).

15. Walsh, P. C., Retnik, A. B., Vaughan, E. D. \& Wein, A. J. Pathophysiology of urinary tract obstruction. Campbell's Urology (7th Ed. Philadelphia) 343-360 (W.B. Saunders Company, 1998).

16. Wagenlehner, F. M. E., Tandogdu, Z. \& Bjerklund Johansen, T. E. An update on classification and management of urosepsis. Curr. Opin. Urol. 27, 133-137 (2017)

17. Kalra, O. \& Raizada, A. Approach to a patient with urosepsis. J. Glob. Infect. Dis. 1, 57-63 (2009).

18. Foxman, B. Urinary tract infection syndromes. Infect. Dis. Clin. N. Am. 28, 1-13 (2014). 
19. Rowe, T. A. \& Juthani-Mehta, M. Urinary tract infection in older adults. Aging Health 9, 519-528 (2013).

20. Nicolle, L. E. Asymptomatic bacteriuria in the elderly. Infect. Dis. Clin. N. Am. 11, 647-662 (1997).

21. Nasa, P., Juneja, D. \& Singh, O. Severe sepsis and septic shock in the elderly: an overview. World J. Crit. Care Med. 1, 23-30 (2012).

22. Hakeem, L. M. et al. Diversity and complexity of urinary tract infection in diabetes mellitus. Br. J. Diabetes Vasc. Dis. 9, 119-125 (2009).

23. Koh, G. C. K. W., Peacock, S. J., van der Poll, T. \& Wiersinga, W. J. The impact of diabetes on the pathogenesis of sepsis. Eur. J. Clin. Microbiol. Infect. Dis. 31, 379-388 (2012).

24. Mnif, M. F. et al. Complicated urinary tract infections associated with diabetes mellitus: pathogenesis, diagnosis and management. Indian J. Endocr. Metab. 17, 442-445 (2013).

25. Kofteridis, D. P. et al. Effect of diabetes mellitus on the clinical and microbiological features of hospitalized elderly patients with acute pyelonephritis: acute pyelonephritis in elderly subjects with DM. J. Am. Geriatr Soc. 57, 2125-2128 (2009).

26. USRDS Annual data report. Atlas of Chronic Kidney Disease in the United States. Available at https://www.usrds.org/2013/pdf/v1_ 00_intro_13.pdf. (2013).

27. Foley, R. N. Infections in patients with chronic kidney disease. Infect. Dis. Clin. N. Am. 21, 659-672 (2007).

28. Tecklenborg, J., Clayton, D., Siebert, S. \& Coley, S. M. The role of the immune system in kidney disease. Clin. Exp. Immunol. 192, 142-150 (2018).

29. Fünfstück, R., Ott, U. \& Naber, K. G. The interaction of urinary tract infection and renal insufficiency. Int. J. Antimicrob. Agents 28, 72-77 (2006)

30. Nahar, A. et al. Pyelonephritis and acute renal failure. Am. J. Med. Sci. 328, 121-123 (2004).

31. Hsiao, C. Y., Yang, H. Y., Hsiao, M. C., Hung, P. H. \& Wang, M. C. Risk factors for development of acute kidney injury in patients with urinary tract infection. PLoS ONE 10, e0133835 (2015).

32. Liang, X. et al. Risk factors and outcomes of urosepsis in patients with calculous pyonephrosis receiving surgical intervention: a single-center retrospective study. BMC Anesthesiol. 19, 61. https://doi.org/10.1186/s12871-019-0729-3 (2019).

33. Hausegger, K. A. \& Portugaller, H. R. Percutaneous nephrostomy and antegrade ureteral stenting: technique-indications-complications. Eur. Radiol. 16, 2016-2030 (2006).

34. Hooton, T. M. et al. Diagnosis, prevention, and treatment of catheter-associated urinary tract infection in adults: 2009 International Clinical Practice Guidelines from the Infectious Diseases Society of America. Clin. Infect. Dis. 50, 625-663 (2010).

35. Chenoweth, C. E. \& Saint, S. Urinary tract infections. Infect. Dis. Clin. North Am. 25, 103-115 (2011).

36. Saint, S., Lipsky, B. A. \& Goold, S. D. Indwelling urinary catheters: A one-point restraint?. Ann. Intern. Med. 137, 125-127 (2002).

37. Chan, J. Y. et al. Predictors of urosepsis in struvite stone patients after percutaneous nephrolithotomy. Investig. Clin Urol. 62, 201-209 (2021).

38. Jeon, C. Y., Neidell, M., Jia, H., Sinisi, M. \& Larson, E. On the role of length of stay in healthcare-associated bloodstream infection. Infect. Control Hosp. Epidemiol. 33, 1213-1218 (2012).

39. Grabe, M. et al. Guidelines on urological infections. European Association of Urology Available at: http://uroweb.org/wp-conte nt/uploads/19-Urological-infections_LR2.pdf. (2015).

40. Shankar, M., Narasimhappa, S. \& Madhura, N. S. Urinary tract infection in chronic kidney disease population: a clinical observational study. Cureus. 13, e12486 (2021).

41. Tandoğdu, Z. et al. Antimicrobial resistance in urosepsis: outcomes from the multinational, multicenter global prevalence of infections in urology (GPIU) study 2003-2013. World J. Urol. 34, 1193-1200 (2016).

42. Gagliotti, C. et al. Escherichia coli and Staphylococcus aureus: bad news and good news from the European Antimicrobial Resistance Surveillance Network (EARS-Net, formerly EARSS), 2002 to 2009. Euro Surveill. 16, 19819 (2011).

43. Saltoglu, N. et al. Comparison of community-onset healthcare-associated and hospital-acquired urinary infections caused by extended-spectrum beta-lactamase-producing Escherichia coli and antimicrobial activities. Int. J. Clin. Pract. 69, 766-770 (2015).

44. Schneeberger, C., Holleman, F. \& Geerlings, S. E. Febrile urinary tract infections: pyelonephritis and urosepsis. Curr. Opin. Infect Dis. 29, 80-85 (2016).

45. Podschun, R. \& Ullmann, U. Klebsiella spp. as nosocomial pathogens: epidemiology, taxonomy, typing methods, and pathogenicity factors. Clin. Microbiol. Rev. 11, 589-603 (1998).

46. Hyun, M., Lee, J. Y., Kim, H. A. \& Ryu, S. Y. Comparison of Escherichia coli and Klebsiella pneumoniae in acute pyelonephritis in Korean patients. Infect. Chemother. 51, 130-141 (2019).

47. Richards, M. J., Edwards, J. R., Culver, D. H. \& Gaynes, R. P. Nosocomial infections in combined medical surgical intensive care units in the United States. Infect. Control Hosp. Epidemiol. 21, 510-515 (2000).

48. Weiner, L. M. et al. Antimicrobial-resistant pathogens associated with healthcare-associated infections: summary of data reported to the National Healthcare Safety Network at the Centers for Disease Control and Prevention, 2011-2014. Infect. Control Hosp. Epidemiol. 37, 1288-1301 (2016).

49. Dedeić-Ljubović, A. \& Hukić, M. Catheter-related urinary tract infection in patients suffering from spinal cord injuries. Bosn. J. Basic Med. Sci. 9, 2-9 (2009).

50. Singer, M. et al. The third international consensus definitions for sepsis and septic shock (Sepsis3). JAMA 315, 801-810 (2016).

\section{Author contributions}

Z.D. participated in the design of the study, performed the statistical analysis, drafted and reviewed the manuscript. G.P. participated in its design and coordination, performed the initial analyses, and helped to draft the manuscript. D.T., B.M. and D.B. participated in the design of the study and acquisition of data. All authors read and approved the final manuscript.

\section{Competing interests}

The authors declare no competing interests.

\section{Additional information}

Correspondence and requests for materials should be addressed to Z.D.

Reprints and permissions information is available at www.nature.com/reprints.

Publisher's note Springer Nature remains neutral with regard to jurisdictional claims in published maps and institutional affiliations. 
(c) (i) Open Access This article is licensed under a Creative Commons Attribution 4.0 International cc) License, which permits use, sharing, adaptation, distribution and reproduction in any medium or format, as long as you give appropriate credit to the original author(s) and the source, provide a link to the Creative Commons licence, and indicate if changes were made. The images or other third party material in this article are included in the article's Creative Commons licence, unless indicated otherwise in a credit line to the material. If material is not included in the article's Creative Commons licence and your intended use is not permitted by statutory regulation or exceeds the permitted use, you will need to obtain permission directly from the copyright holder. To view a copy of this licence, visit http://creativecommons.org/licenses/by/4.0/.

(C) The Author(s) 2021 